Vol. $7 \mid 20$ | International Journal of

№ 2120 Medical and Surgical Sciences

\begin{tabular}{l||l|l|l|l}
\hline UA & UNIVERSIDAD \\
AUTONOMA \\
DE C H I L E
\end{tabular}

Case Report

Manejo de Linfoma PRIMARIO de BAZO EN PACIENTE CON VIH-SIDA: REPORTE DE GASO

\title{
Management of primary lymphoma of the spleen in a patient with HIV-AIDS: Case report
}

\author{
HÉCtor Vergara-Miranda (i) \\ Universidad Autónoma de Nuevo León, Hospital Universitario "Dr. José Eleuterio González" Departamento de \\ Cirugía General, Monterrey, Nuevo León, México.
}

Luis Adrian Alvarez-Lozada (1)

Universidad Autónoma de Nuevo León, Facultad de Medicina, Grupo Estudiantil con Interés en el Campo

Quirúrgico, Monterrey, Nuevo León, México.

\section{Carlos Pacheco-Molina (i)}

Universidad Autónoma de Nuevo León, Hospital Universitario “Dr. José Eleuterio González" Departamento de

Cirugía General, Monterrey, Nuevo León, México.

\section{Alejandro Quiroga-Garza}

Universidad Autónoma de Nuevo León, Facultad de Medicina, Grupo Estudiantil con Interés en el Campo

Quirúrgico, Monterrey, Nuevo León, México.

Universidad Autónoma de Nuevo León, Facultad de Medicina, Departamento de Anatomía Humana, Nuevo León, México.

Instituto Mexicano del Seguro Social, Monterrey, Nuevo León, México.

Autor de correspondencia: Dr. Alejandro Quiroga-Garza

Departamento de Anatomía Humana, Facultad de Medicina, Universidad Autónoma de Nuevo León.

Ave. Madero y Aguirre Pequeño, Col. Mitras Centro, s/n, Col.

Mitras Centro, Monterrey, Nuevo León, México, C.P. 64460

Correo electrónico: dr.aquirogag@gmail.com

Receipt: $19 / 08 / 2020$

Acceptance: 06/10/2020

\section{RESUMEN}

Los linfomas primarios de bazo afectan al bazo y/o sus ganglios linfáticos inicialmente. Representan $<1 \%$ de los linfomas e implican un reto diagnóstico, por baja prevalencia y sintomatología inusual. Por lo tanto, el objetivo de este trabajo fue describir el manejo y evolución de un paciente con VIH-SIDA y la presentación de un Linfoma primario de bazo.

En este reporte presentamos el caso de un paciente masculino de 52 años con VIH sin apego a tratamiento debuta con dolor abdominal, fiebre y pérdida de peso de 2 meses de evolución. La tomografía computarizada properatoria revela esplenomegalia y lesiones hipodensas sospechosas 
de neoformación. Es intervenido, realizándose una laparotomía exploratoria, con esplenectomía. El estudio histopatológico reporta linfoma no Hodgkin.

El linfoma primario de bazo es poco frecuente. Es necesario su conocimiento para su sospecha diagnóstica y un diagnóstico diferencial en pacientes con esplenomegalia.

Palabras clave. Linfoma; bazo; esplenectomía.

\section{Introducción}

El linfoma es una proliferación clonal de los linfocitos, lo que deriva de una activación de un receptor en específico de los linfocitos, por un antígeno en particular, que provoca que se diferencien en más células capaces de reconocer a este antígeno, produciendo una proliferación de un solo tipo celular. Se dividen en dos principales categorías, Linfomas Hodgkin y Linfomas no-Hodgkin (LNH). Estos últimos, representan un conjunto de neoplasias que pueden derivar de los linfocitos B y $\mathrm{T}$. Tienen la capacidad de desarrollarse en cualquier tejido que presente células de la estirpe linfoide al estar expuestos a antígenos, como el bazo, médula ósea, timo y otros órganos.(Carvajal et al., 2007; Más et al., 2013).

Los linfomas primarios de bazo (LPB) son una presentación infrecuente del cáncer. Representan $<1-2 \%$ de todos los linfomas (Carvajal et al., 2007; Kurzepa et al., 2017). Puede manifestar datos de esplenomegalia, afectación desde una a tres estirpes celulares, generalmente con adenopatías al momento del diagnóstico y metástasis a distancia, como hígado, ganglios locales o regionales y en médula ósea. .(Carvajal et al, 2007; Más et al., 2013).

Dasgupta et al. (1965) establecieron una definición aún vigente para los LPB, establecida como una neoplasia del subtipo no Hodgkin, que involucra el bazo y sus ganglios hiliares, con un período libre de enfermedad de al menos 6 meses, después de la extirpación quirúrgica del bazo (Dasgupta et al., 1965). Sin embargo, no todos los reportes incluyen esta definición, por lo que la prevalencia exacta del LPB es incierta (Cotta-Pereira et al., 2011).

Debido a que la fisiopatología responde a una proliferación clonal secundaria a una exposición a un antígeno en específico, Cotta et al. (2011) han establecido una posible correlación con el virus de la hepatitis $\mathrm{C}(\mathrm{VHC})$ como un partícipe importante en el desarrollo de la proliferación (Cotta-Pereira et al., 2011).

El objetivo de este reporte es describir el manejo y evolución de un paciente con VIH-SIDA y la presentación de un Linfoma primario de bazo, discutiendo los retos de su diagnóstico debido a la presentación clínica inusual.

\section{Caso clínico}

Paciente masculino de 52 años acude a Urgencias por dolor abdominal. Refiere antecedentes de infección por virus de inmunodeficiencia humana $(\mathrm{VIH})$ de dos años de diagnóstico con mal apego a tratamiento. Pérdida de peso $>20 \%$, astenia, adinamia e hiporexia de 2 meses de evolución. Acude por dolor abdominal tipo punzante en hipocondrio izquierdo, de 72 horas de evolución acompañado de episodios febriles $>38^{\circ} \mathrm{C}$. 
Durante su exploración física, temperatura de $38.3^{\circ} \mathrm{C}$, no se identifican adenopatías periféricas visibles ni palpables, con abdomen blando depresible, doloroso a la palpación en hipocondrio izquierdo con hallazgos clínicos de esplenomegalia palpable $4 \mathrm{~cm}$ debajo de reborde costal. Estudios de laboratorio reportan hemoglobina $6.45 \mathrm{~g} / \mathrm{dl}$, hematocrito $21.7 \%$, volumen corpuscular medio $77.4 \mathrm{fl}$, leucocitos $4.79 \mathrm{~K} / \mathrm{uL}$, plaquetas $277 \mathrm{~K} / \mathrm{uL}$, y DHL $275 \mathrm{UI} / \mathrm{L}$. Hemocultivo, urocultivo y Ziehl-Nielsen de esputo negativos. Serología VDRL (+) 1:4, AntiAc anti VIH1/VIH2 (+) 798.12 S/ $\mathrm{CO}$, perfil viral para hepatitis $\mathrm{B}, \mathrm{C}$, citomegalovirus y Epstein-Barr negativos. Radiografía de tórax sin evidencias de patología. Tomografía computarizada (TG) abdominal con contraste intravenoso evidencia esplenomegalia $(20 \times 16 \times 15 \mathrm{~cm})$ con imágenes hipodensas en su interior de $6 \times 7 \times 6 \mathrm{~cm}$ con sospecha diagnostica de un proceso linfoproliferativo versus abscesos esplénicos. (Figura 1)

\section{Figura 1.}

Estudio de tomografía computarizada demostrando bazo aumentado de tamaño con múltiples lesiones lobuladas dentro de su parénquima. (A) corte axial; (B) reconstrucción sagital; $(\mathrm{C})$ reconstrucción coronal.

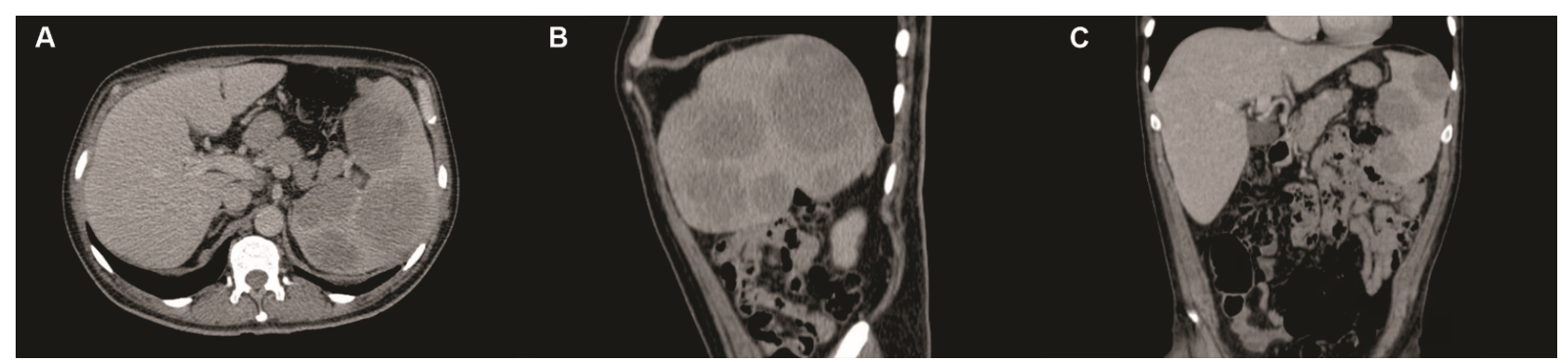

Debido a la limitación de recursos del paciente para una biopsia guiada por imagen, se decide proceder directo a manejo diagnóstico y terapéutico mediante esplenectomía abierta mediante laparotomía supraumbilical. El bazo se visualiza de $21 \times 13 \times 9 \mathrm{~cm}$, con pérdida de su morfología a expensas de aumento de volumen por múltiples lesiones lobuladas, induradas, las cuales protruyen a través de la capsula. (Figura 2). No se evidencian adenopatías regionales, mesentéricas o paraaórticas, líquido libre, ni lesiones hepáticas. Se realiza esplenectomía realizando apertura de trascavidad de los epiplones, se liga y secciona epiplón gastrocólico, se desplaza el estómago hacia cefálico, se realiza

Figura 2.

Muestra patológica macroscópica de bazo post-esplenectomía. (A) pieza completa mostrando distorsión de bazo, con lesiones que protruyen de la cápsula; (B) sección de bazo demostrando lisis de tejido y perdida arquitectural del órgano. (G) lesiones firmes, lobuladas, bien delimitadas

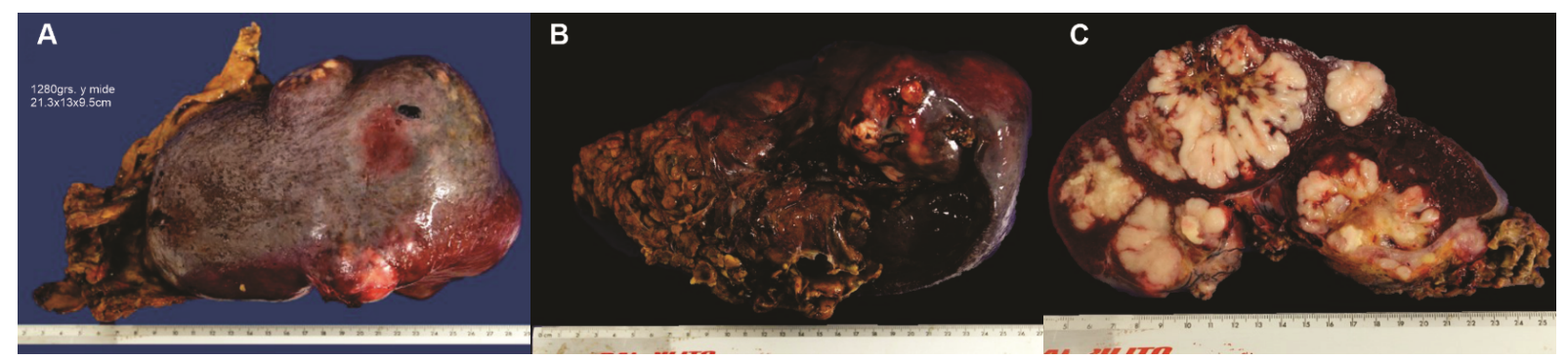


disección y control de arteria esplénica en el hilio, se realiza apertura de ligamento gastroesplénico con ligadura no absorbible y sección de vasos cortos, se dividen los ligamentos esplenocólico, esplenorrenal y esplenofrénico aproximando el bazo a la superficie; se diseca y secciona arteria y vena esplénica, extirpando el bazo. Se verificó hemostasia con sangrado de 200cc cerrando por planos con cuidados y prevención de herida (Quiroga-Garza et al., 2017). Paciente presenta adecuada recuperación postquirúrgica, con manejo analgésico con AINES vía oral con escala de EVA menor a 3, tolerando dieta a las 24 horas del postquirúrgico y con profilaxis antibiótica con cefalotina 1 gr IV cada 8 horas hasta su egreso al tercer día postquirúrgico.

Paciente con adecuada evolución en citas de seguimiento por cirugía general, con adecuada evolución de herida quirúrgica sin datos de infección o dehiscencia, con reincorporación a las actividades cotidianas a los 21 días del postquirúrgico. Sin evidencia de enfermedad sistémica o recurrencia asociada a linfoma dentro los primeros 3 meses. En seguimiento y vigilancia actual por servicio de Hematología.

El estudio histopatológico reporta 8 lesiones de aspecto lobulado y firmes, de bordes bien definidos respecto al resto del parénquima esplénico (Figura 2). Microscopia muestra perdida de la arquitectura normal del órgano, por infiltración de células pleomórficas linfoides formada por células de gran tamaño y presencia de actividad mitótica. Se complementa con técnica de FISH la que revela positividad para CD20 y MUM-1, concluyendo como Linfoma No Hodgkin Difuso de Células Grandes B Post Germinal. Se realizaron marcadores de inmunohistoquímica, los cuales se interpretaron de la siguiente manera: CD20 positivo, CD30 negativo, MUM1: positivo, ALK negativo. (Figura 3)

Figura 3.

A, B (CD20 positivo), C (CD30 negativo), D (MUM1 positivo).

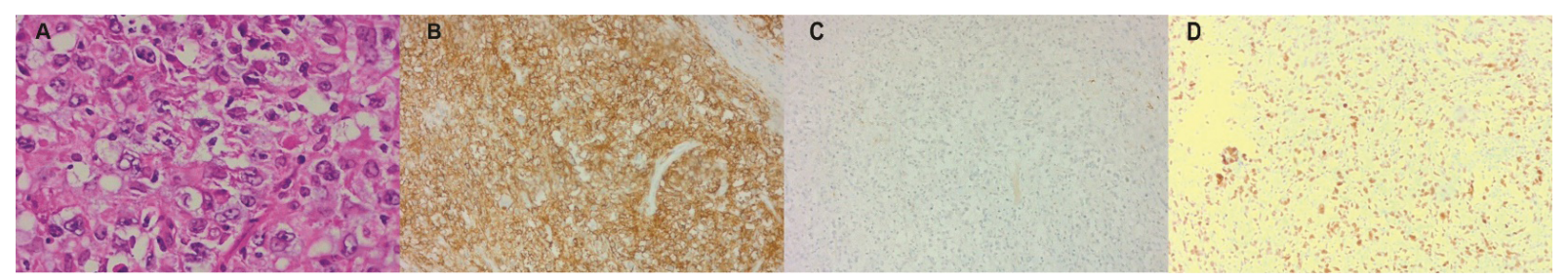

El presente artículo se presenta siguiendo las guías CARE, bajo consentimiento informado del paciente implicado, con los principios de confidencialidad para manejo de información y garantizando sus derechos, con la finalidad de mejorar el diagnóstico, manejo y evolución de los pacientes con dicha patología.

\section{Discusión}

Los LPB son una presentación atípica de las neoplasias de linfomas y representan un desafío en reconocerlas y diagnosticarlas, por distintas razones, el clínico debe tener conocimiento de la patología para sospecharla, aunque debido a su baja prevalencia y sus escasos reportes dificultan el proceso. Asimismo, su sintomatología tiende a ser inespecífica y puede diseminarse rápidamente.(Carvajal et al., 2007; Izzo et al., 2002)

Clínicamente se caracteriza por tener síntomas de esplenomegalia, aunque generalmente cursa asintomático hasta encontrarse en estadios avanzados y genere síntomas inespecíficos como dolor, 
pérdida de peso, anorexia, astenia, adinamia, fiebre, palidez o diaforesis nocturna.(Carvajal et al., 2007; Antón et al., 2004)

Puede también asociarse con anemia, trombocitopenia o leucopenia manifestando pancitopenia, en especial durante fase aguda y LDH. Un 40\% de los casos puede debutar con linfocitosis. (Carvajal et al., 2007; Más et al., 2013; Izzo et al., 2002) Se relaciona con mayor frecuencia al género femenino, con su principal prevalencia entre la segunda y cuarta década de vida.(Carvajal et al., 2007; CottaPereira, 2011)

Cotta et al. han propuesto su posible origen involucrando al VHC, explicado por su linfotropismo viral, que provoca una expansión monoclonal o policlonal de los linfocitos. Los antígenos del VHC despiertan una respuesta inmunológica con una estimulación intensa de los Linfocitos B.(CottaPereira, 2011)

El diagnóstico debe complementarse con estudios de imagen. El ultrasonido (US) es el estudio inicial de elección. Identifica la esplenomegalia y lesiones de diferente ecogenicidad. Permite demarcar una lesión linfoide, con su tejido esplénico circundante y proporciona información útil sobre los diagnósticos diferenciales entre una lesión esplénica benigna o maligna. Sin embargo un tamaño y aspecto normal, no excluye un linfoma. (Kurzepa et al., 2017)

La Tomografía Computarizada (TG) se prefiere si el US no fue concluyente. Estos ayudan en la correcta visualización de la lesión esplénica, así como a definir sus características e invasión extraesplénica en caso de presentarlo. Se pueden identificar 4 patrones radiológicos mencionados por Carvajal et al. (2007): 1) Esplenomegalia homogénea, 2) patrón miliar con nódulos entre 1-5 mm, 3) Masas de tamaño heterogéneo, entre 2-10 mm, 4) una masa de entre 7-14 cm de diámetro, con o sin hipodensidad central (Carvajal et al., 2007).

Otros estudios de utilidad incluyen la resonancia magnética la cual ofrece mayor resolución, y la tomografía por emisión de positrones (PET) que permite identificar pequeños focos de enfermedad y actividad en masas residuales (Carvajal et al., 2007).

Debido a la presentación clínica de dolor abdominal punzante y por la localización específica, obliga a sospechar la presencia de una patología esplénica. Por la localización del dolor, uno de los diagnósticos diferenciales, aunque infrecuentes, es un infarto esplénico. Dicha patología suele tener una instalación de dolor súbita, además de presentarse con infecciones de base, lo que no es infrecuente a la patología asociada de infección por VIH, considerando su pobre control terapéutico. En la TC contrastada suele mostrar una hipodensidad característica en cuña dirigida hacia el hilio esplénico, la cual no presentaba el paciente en cuestión, por lo que pudo descartarse esta situación (Chapman et al., 2017). Otra sospecha diagnóstica sería tumoraciones de diversas etiologías, incluyendo infecciosa (absceso esplénico). Esta se manifestaría como áreas hipodensas delimitadas en la TC. Se debe sospechar así mismo de infecciones bacterianas o alteraciones de la colágena.( Martínez et al., 2001). La sospecha de absceso esplénico era fuerte debido a los episodios febriles y la clínica de ataque al estado general, solo pudo ser descartada una vez enviada la muestra a patología.

La confirmación del diagnóstico es mediante estudio histopatológico, inmunohistoquímica y genotipificación. Se prefiere biopsia guiada por imagen, previo a establecer el tratamiento quirúrgico. (Carvajal et al., 2007; Más et al., 2013; Pirovano et al., 2019). En nuestro caso, por la falta de recursos económicos lo cual fue una limitante del paciente, se decide realizar la esplenectomía sin previa biopsia para resolución del padecimiento. Los estudios de imagen demostraban ausencia de adenopatías locales o a distancia. Siempre deben tomarse en consideración ciertos parámetros para realizar la esplenectomía como método diagnóstico: grado de esplenomegalia, tamaño de la tumoración 
esplénica y si la sintomatología representa un problema que interfiere en la vida del paciente (CottaPereira et al., 2011; Izzo et al., 2002)

Un período de 6 meses libre de la enfermedad confirma el diagnóstico. La esplenectomía y la erradicación de las masas neoplásicas determinan la remisión de la sintomatología del paciente y en la mayor parte de los casos resuelve las citopenias que se hayan podido desarrollar en el curso de la patología (Carvajal et al., 2007; Izzo et al., 2002). En pacientes con LNH de bajo grado confinado al bazo, la remisión puede ser completa o parcial de las alteraciones hematológicas asociadas, y puede alcanzarse aproximadamente a los siete meses de la esplenectomía.(Carvajal et al., 2007)

El tratamiento neoadyuvante es controversial (Cotta-Pereira et al., 2011; Izzo et al., 2002). Existen estudios que reportan que no hay diferencia significativa entre manejo con quimioterapia versus cirugía en el caso de LPB (Izzo et al., 2002). La radioterapia puede ayudar en casos avanzados, para disminuir la extensión tumoral y el tamaño del bazo, pero mejora los parámetros hematológicos (Carvajal et al., 2007)

La morbilidad postoperatoria es del 1.5-50\% de los casos, se presenta con neumonía, hemorragia digestiva, trombosis venosa profunda, del sistema portal y el sistema cava, fallo hepático, infección de la herida, abscesos, fístulas pancreáticas, etc. (Carvajal et al., 2007; Chapman et al., 2017; Martínez et al., 2001). El síndrome sepsis grave post esplenectomía es una de las complicaciones más temidas en la evolución postoperatoria de las esplenectomías, debido al déficit inmunológico que este procedimiento conlleva. Se trata de una infección de progresión aguda en la que se desarrolla bacteriemia con Streptococcus pneumoniae en el 50\% de los casos. Se desarrolla del 30-50\% en los primeros dos años posteriores a la operación y tiene una mortalidad del 50-80\% (CENETEC, 2010; Buzelé et al., 2016).

Debido a que el bazo tiene una función inmunológica específica para bacterias encapsuladas, contenida en la zona marginal en donde se encuentran los linfocitos B que poseen memoria, el cuerpo es especialmente susceptible ante estos patógenos una vez este órgano es extirpado. Los principales patógenos a los que el cuerpo es susceptible de agresión son Streptococcus pneumoniae, Neisseria meningitidis y Haemophilus influenzae B, todas bacterias encapsuladas. Ante la víspera de una esplenectomía, se debe aplicar inmunización contra estos tres agentes al menos con dos semanas de antelación, para generar la respuesta inmune. En caso de que esto no sea posible, debe aplicarse dos semanas posteriores, debido a que la respuesta no será efectiva en estas dos primeras post esplenectomía. Se recomienda asimismo la inmunización anual contra influenza (CENETEC, 2010; Buzelé et al., 2016).

Un punto fuerte de este caso es su presentación inusual. Los cirujanos deben tener en mente diagnósticos diferenciales como linfoma, en paciente de alto riesgo $(\mathrm{VIH})$ y esplenomegalia de etiología desconocida. El caso fue limitado por los recursos del paciente, motivo por el cual el tratamiento quirúrgico se establece, previo a un diagnóstico certero. Una biopsia guiada por un método de imagen sería la primera opción. El estudio histopatológico es de vital importancia, para establecer el manejo a continuar en pacientes como este y establecer un mejor pronóstico.

\section{Conclusión}

El linfoma primario de bazo es una entidad poco frecuente y su diagnóstico y tratamiento presentan un reto importante. Se presenta el caso clínico de un paciente con dolor abdominal en el que se diagnóstica esplenomegalia. El antecedente de VIH-SIDA es importante en la etiología del padecimiento. El tratamiento de esplenectomía fue favorable para la evolución del paciente. 
Aunque su incidencia es baja, los cirujanos deben tener en mente este padecimiento como diagnóstico diferencial en casos similares.

\section{Agradecimientos}

Ofrecemos nuestro sincero agradecimiento a la Facultad de Medicina de la Universidad Autónoma de Nuevo León, así como al Hospital Universitario Dr. José Eleuterio González, al Departamento de Cirugía General y a su personal por su cooperación en la realización de este artículo. Asimismo, agradecemos al Dr. Alejandro Quiroga Garza por su asesoramiento en la redacción y constitución del presente artículo y a Luis Adrian Alvarez Lozada por la redacción de este.

\section{Conflicto de Intereses}

Los autores declaran que no existen conflictos de intereses para desarrollar el trabajo ni para publicarlo entre los proponentes y los participantes en este trabajo.

\section{Financiamiento}

Estudio de investigación no sujeto financiamiento.

\section{Referencias}

Carvajal, J., Simón, L., Oliart, S., Martín, M., Camuñas, J., Peña, L., et al. (2007). Linfoma primario de bazo de presentación asintomática. Mapfre Medicina, 18(3):219-24. https://sid.usal.es/idocs/ F8/ART9902/linfoma.pdf

Más, J., Rodríguez, M., Cuellar, A., G, A., \& Más, R. (2013). Linfoma primario de bazo. Presentación de un caso. Gaceta Médica Espirituana, 15(3):317-23, http://revgmespirituana.sld.cu/index.php/ gme/article/view/329/html_4

Cotta-Pereira, R., Acar, R., Mello, C. E., Iglesias, A. G., \& Basílio-de-Oliveira, G. A. (201 1). Primary spleen lymphoma associated with hepatitis $\mathrm{C}$ virus infection. BMJ case reports, https://doi. org/10.1136/bcr. 11.2010 .3474

Izzo, Luciano \& Binda, Barbara \& Boschetto, Arianna \& Caramanico, Luciano \& Galati, Gaspare \& Fiori, Enrico \& Mele, Luigi \& Stasolla, Alessandro. (2002) Primitive spleen lymphoma: diagnostic and therapeutic value of splenectomy. Haematologica. Vol. 87 No. 6. https://doi.org/10.3324/\%25x

Antón, E., Vidal, M.J., \& Lobo, C. (2004) An unusual presentation of primary malignant lymphoma of the spleen. American Journal of Hematology, 77(4), 416-17. https://doi.org/10.1002/ajh.20170

Kurzepa, J., Uhlig, S., Czekajska-Chehab, E., Siek, E., Zakościelna, M., Drop, A. (2017) Primary spleen lymphoma visualized with contrast enhanced ultrasound - Case report. Journal of PreClinical and Clinical Research, 11(2):157-59. https://doi.org/10.26444/jpccr/80919

Dasgupta, T., Coombes, B., \& Brasfield, R. D. (1965) Primary malignant neoplasms of the spleen. Surg Gynecol Obstet.120:947-960. 
Quiroga-Garza, A., Valdivia-Balderas, J. M., Trejo-Sánchez, M. Á., Espinosa-Uribe, A. G., ReyesHernández, C. G., \& Elizondo-Omaña, R. E.(2017). A Prospective, Randomized, Controlled Clinical Trial to Assess Use of 2\% Lidocaine Irrigation to Prevent Abdominal Surgical Site Infection. Ostomy Wound Management, 63(8):12-21. https://doi.org/1025270/owm.2017.08.1221

Chapman, J., Helm, T. A., \& Kahwaji, G. I. (2017) Splenic Infarcts. In StatPearls. StatPearls Publishing https://www.ncbi.nlm.nih.gov/books/NBK430902/

Martínez, C., Esparcia Navarro, A., Roig Rico, P., \& Cañizares, R.(2001) Linfoma no Hodgkin Esplénico. Aportación de un nuevo caso y revisión de la literatura. Revista Clínica Española, 201(8):494-495. https://doi.org/10.1016/s0014-2565(01)70894-6

Pirovano, A., Matino, E., Zecca, E., Costanzo, M., Croce, A., Leutner, M., et. al. (2019) A 28-YearOld Woman with Ascites and Multiple Focal Spleen Lesions. European Journal of Case Reports in Internal Medicine, 6(5) https://doi.org/10.12890/2019_001061.

CENETEG (2010) Guía de practica clinica - esplenectomia SSA-301-10; Mexico: Secretaria de Salud.

Buzelé, R., Barbier, L., Sauvanet, A., Fantin, B.(2016) Medical complications following splenectomy. Journal of Visceral Surgery,153(4):277-86. http://dx.doi.org/10.1016/j.jviscsurg.2016.04.013

\begin{abstract}
Primary splenic lymphomas involve the spleen and/or its lymph nodes. They represent $<1 \%$ of lymphomas and they imply a diagnostic challenge due to its poor symptomatology and low prevalence.

There for, the objects this work describe the management and evolution of a patient with HIVAIDS and presentation of a primary lymphoma of the spleen

In this report we present the case of a 52-year-old male patient with HIV infection without treatment presented with abdominal pain, fever and a 2-month weight loss. The pre-operative computed tomography showed splenomegaly and hypodense lesions suspected to be tumoral. He was operated on, performing an exploratory laparotomy, performing an splenectomy. The histopathological study reported a non-Hodgkin lymphoma.

Primary splenic lymphoma is a non frequent entity. It is necessary to be acquainted with it in order to suspect it.
\end{abstract}

Key words. Lymphoma; spleen; splenectomy. 